\title{
A Study of Y Chromosome Microdeletions in Infertile Indian Males
}

\author{
Arundhati S. Athalye, Prochi F. Madon, Nandkishor J. Naik, Dattatray J. Naik, Smita S. Gavas, \\ Suresh B. Dhumal, Vijay M. Bandkar, Mahadev T. Kawle and Firuza R. Parikh \\ Department of Assisted Reproduction and Genetics, Jaslok Hospital and Research Center, \\ 15, Dr. G. Deshmukh Marg, Mumbai 400 026, Maharashtra, India \\ E-mail:prochimadon@hotmail.com
}

KEYWORDS Male infertility; ICSI; semen analysis; PGD; AZF deletions; azoospermia; OAT

\begin{abstract}
Male partners of infertile couples are known to frequently have abnormal semen parameters. Some of these cases are due to underlying genetic factors such as $\mathrm{Y}$ chromosome microdeletions, an abnormal karyotype or cystic fibrosis mutations. Y chromosome microdeletions generally cannot be detected by karyotyping. At our clinic we undertook a study of male partners of infertile couples to determine the frequency and common loci of $\mathrm{Y}$ chromosome microdeletions in India, using the PCR technique. We studied 100 patients mainly having azoospermia (AZ) or oligoasthenoteratozoospermia (OAT). Multiplex PCR analysis for 18 loci on the Y chromosome was carried out using commercially available kit (Promega Version 1.1). Y chromosome microdeletions were observed in 12/100 (12\%) patients including $8 / 27(29.63 \%)$ with azoospermia, 3/56 (5.35\%) with oligoasthenoteratozoospermia and 1/ $7(14.28 \%)$ with only asthenoteratozoospermia. All loci of the DAZ gene were deleted along with DYS237 and DYS236 from AZFd in 5/27 (18.52\%) azoospermic males studied. The most commonly deleted loci were DYS240 in $11 / 12(91.67 \%)$ and DYS219 in 7/12 (58.33\%) patients with microdeletions. The use of ICSI in such patients can lead to transmission of $\mathrm{Y}$ chromosome microdeletions and subsequent infertility from father to son. Hence screening for $\mathrm{Y}$ chromosome microdeletions will help in the proper counseling and management of couples with male factor infertility.
\end{abstract}

\section{INTRODUCTION}

A significant proportion of infertile males present with oligoasthenoteratozoospermia (OAT) or azoospermia (AZ). Alterations in spermatogenesis can be the consequence of many causes, such as systemic diseases, cryptorchidism, endocrinological factors, infections and obstruction/absence of seminal pathways. There is evidence that $60 \%$ cases of male infertility have an underlying genetic basis (Lilford et al. 1994). Y chromosome microdeletions are small deletions in the distal euchromatic region of the long arm of the $\mathrm{Y}$ chromosome, in intervals 5 and 6 (Vergnaud et al. 1986). This region mainly comprises the azoospermia factors - AZF a, b, d and c. Genes like DAZ (Deleted in Azoospermia) and RBM (RNA Binding Motif) have proved to be of particular interest in this region and occur in multiple copies (Raman 1999). Search for Y chromosome microdeletions is essentially focused on screening the AZF region through STSs (sequence tagged sites) spanning this area.

The advent of intracytoplasmic sperm injection (ICSI) allows the possibility of biological fatherhood to many couples with male factor infertility. However, this raises the possibility of transmission of genetic defects to the son in cases where the male partner has a genetic abnormality such as Y chromosome microdeletions. In order to determine the cause of male infertility and to provide proper counseling, the clinical investigation should include semen analysis, karyotyping and a DNA analysis to rule out $\mathrm{Y}$ chromosome microdeletions. If the cause of infertility is known, then transmission of genetic abnormalities to the offspring can be minimized by offering preimplantation genetic diagnosis (PGD).

\section{Y Chromosome Microdeletion}

The $\mathrm{Y}$ chromosome is not essential for life. Until recently most regions of the $\mathrm{Y}$ chromosome were assumed to be functionally inert. Sex determination controlled by the SRY gene was viewed as the sole function related to the $\mathrm{Y}$ chromosome. However, in recent years, the control of spermatogenesis was found to be 
associated with the $\mathrm{Y}$ chromosome and many genes have been mapped to the $\mathrm{Y}$ chromosome (Foresta et al. 2001).

Involvement of the $\mathrm{Y}$ chromosome in male infertility was originally suggested by cytogenetically detectable terminal deletions of the long arm of the $\mathrm{Y}$ chromosome, in which the entire Yq heterochromatic region was lost (Tiepolo et al. 1976). This type of deletion does not appear to impair spermatogenesis in some men, but leads to infertility in others. The simple explanation for these observations was the presence of a key locus (or loci) close to the boundary between $\mathrm{Yq}$ euchromatin and genetically inert heterochromatin (Borgaonkar et al. 1971). In some men, the removal of this locus by more-extensive deletions, which could not be observed by cytogenetic analysis, causes infertility. Infertile men may also have small deletions in other regions of the Y chromosome. The overall frequency of $\mathrm{Y}$ chromosome microdeletions has been estimated to be $12.2 \%$ in azoospermic men and $3.4 \%$ in oligozoospermic men (Simoni et al. 1998).

Definitive genetic evidence that loci on the long arm of the human $\mathrm{Y}$ chromosome are required for the differentiation of germ cells has come from studies using molecular markers, such as STSs that have been mapped at high density along the Y chromosome. At least three distinct nonoverlapping regions, each associated with variable degrees of spermatogenic impairment, have been defined by numerous deletionmapping studies in the Azoospermia Factor (AZF) region. These regions named as AZFa, $\mathrm{AZFb}$ and $\mathrm{AZFc}$ for azoospermia factors $\mathrm{a}, \mathrm{b}$ and $\mathrm{c}$ indicate that at least three different loci on the long arm of the $\mathrm{Y}$ chromosome are critical for germ-cell differentiation. A fourth locus AZFd has been suggested afterwards which lies between loci AZFb and AZFc (Vogt et al. 1992; Vogt et al. 1996; Pryor et al. 1997; Elliott et al. 1997; Kent-First et al. 1999).

Early studies attempted to assign specific infertility phenotypes to each region. However Pryor et al. (1997) showed that men with mild oligozoospermia and those with normal sperm counts but abnormal sperm morphology can have microdeletions in either AZFa, AZFb or AZFc loci. Ferlin et al. (2003) also did not find any relationship between the genotype and phenotype in their study, which they hypothesize, may be due to additional genetic and environmental factors. Although a definite genotype-phenotype correlation is not known for Yq microdeletions, it is generally accepted that large deletions that span multiple AZF regions or those restricted to AZFa usually result in Sertoli cell-only syndrome or severe oligozoospermia (Vogt et al. 1996). Microdeletions restricted to $\mathrm{AZFb}$ or $\mathrm{AZFc}$, can result in a range of phenotypes from Sertoli cell-only syndrome to moderate oligozoospermia. Those restricted to the AZFd region may present with mild oligozoospermia or even normal sperm counts, with abnormal sperm morphology (Vogt et al. 1996; Pryor et al. 1997; Foresta et al. 1998; KentFirst et al. 1999). In India, Raman (1999) was one of the first to work on Y microdeletions. Babu et al. (2002) studied 20 infertile males from South India and found $15 \%$ AZF deletions in azoospermic and severely oligozoospermic men. Dada et al. in 2003 reported the frequency of $Y$ microdeletions to be $9.63 \%$ among 83 infertile Indian males studied using 6 STS primers. In another study from South India (Swarna et al. 2003) reported 4/50 infertile males with AZFc deletions. The largest Indian study of Y chromosome deletions in azoospermic men was by Thangraj et al. (2003).

The Deleted in Azoospermia (DAZ) gene family is reported to be the most frequently deleted AZF candidate gene and is located in the AZFc region (Reijo et al. 1995). The DAZ genes are expressed exclusively in testicular tissue and encode proteins that contain an RNArecognition motif, thereby suggesting that they have a regulatory role in RNA metabolism (Habermann et al. 1998). This would mean that all men with DAZ deletions would be incapable of producing mature sperm (Kent-First et al. 1996). However, some men with oligozoospermia have been shown to carry DAZ deletions (Kent-First et al. 1999).

There is paucity of data on $\mathrm{Y}$ chromosome microdeletions in the Indian population, especially from western India. The present study was undertaken to determine the frequency and find common loci of $\mathrm{Y}$ chromosome microdeletions among infertile men in India. An attempt was made to correlate the genotype and phenotype in these cases by comparing results of semen analysis with $\mathrm{Y}$ chromosome microdeletions. We also wanted to evaluate any variation in the incidence of $\mathrm{Y}$ chromosome microdeletions in Indian infertile males as compared to Western countries. 


\section{MATERIALANDMETHODS}

Selection of Samples: The study population of 100 males was selected from patients visiting our infertility clinic at Jaslok Hospital and Research Center in Mumbai, India with a history of male factor infertility. Semen analysis is routinely performed on the male partner of couples coming for infertility treatment. The semen samples were analyzed for sperm-count, motility, viability and morphology. The 100 patients were categorized into two main groups for this study. One group consisted of 27 patients with azoospermia (AZ), while the other group of 73 patients broadly classified as OAT, consisted of 56 patients with oligoasthenoteratozoospermia, 7 with asthenoteratozoospermia, 5 with oligoteratozoospermia, 4 with oligozoospermia and 1 with teratozoospermia. All 100 patients were analyzed for $Y$ chromosome microdeletions together with five known fertile males who were taken as controls. Semen Analysis: Manual semen analysis was performed using Makler counting chamber (Sefi Medical Instruments, USA) and Kruger strict morphology criteria were used to categorize the type of abnormality in the semen sample.

Y Chromosome Microdeletion Detection: Eighteen microdeletions on the $\mathrm{Y}$ chromosome were analyzed by multiplex PCR using commercially available kits (Y Chromosome Deletion Detection System, Version 1.1, Promega). All the primer pairs were homologous to previously identified and mapped STSs that were separately located in AZFa, AZFb, AZFc and AZFd regions of Yq. All primers were nonpolymorphic short DNA fragments grouped into 4 sets as multiplex A, B, C and D. This enabled the determination of the presence or absence of all 18 STSs by performing a one-time PCR amplification to identify Y chromosome deletions in these regions associated with male infertility, as per the manufacturer's instructions. The internal positive control in each multiplex was the SMCX locus on the $\mathrm{X}$ chromosome.

For PCR, 2-3 ml venous blood was collected in EDTA. DNA was extracted using commercial extraction kits (Qiagen, Amersham Pharmacia Biotech or Promega). The PCR program used was one cycle at $94^{\circ} \mathrm{C}$ for 10 minutes followed by 35 cycles of $94^{\circ} \mathrm{C}$ for 1 minute, $57^{\circ} \mathrm{C}$ for 30 seconds and $72^{\circ} \mathrm{C}$ for 1 minute. This was followed by one extension cycle at $72^{\circ} \mathrm{C}$ for 10 minutes. The PCR products were separated by electrophoresis on
2.5\% agarose gel. A 50bp DNA ladder was loaded with PCR products to estimate band size. The gel was stained with ethidium bromide and photographed.

\section{RESULTS}

$\mathrm{Y}$ chromosome microdeletions were observed in $12 / 100(12 \%)$ infertile patients studied. A comparison of the number and percentage of cases with Y chromosome microdeletions in the $\mathrm{AZ}$ and OAT groups is given in Table 1. Microdeletions were present in 8/27 (29.63\%) patients with azoospermia and 4/73 (5.48\%) patients in the OAT group. Table 2 shows the actual deletions of STSs and related AZF regions in each of the 12 cases. The highest number of loci deleted [(16/18) i.e. $88.89 \%$ ], were observed in 2 patients (Yd068 and Yd097). Only 2 loci DYS215 and DYS223 in the AZFb region were not deleted in one patient (Yd068). The other patient (Yd097) showed that only DYS271 and KALY from the AZFa region were not deleted. Cytogenetic abnormalities also detected in these 2 cases will be described elsewhere. Of the 18 STSs analyzed, the number of STSs found to be deleted per patient ranged from $1 / 18$ to $16 / 18$ with a mean of $5.36 \pm 4.38$. The highest number of patients with microdeletions (29.63\%) observed in our study was in the group of 27 males with azoospermia. The most common locus deleted in our study was DYS240 (SY157) from the AZFc region in 11/12 (91.6\%) patients with $\mathrm{Y}$ chromosome microdeletion/s. The next common locus deleted was DYS219 (SY128) from the AZFb region in 7/12 (58.3\%) patients. Four of these patients also showed deletion of DYS212 (SY121) from the $\mathrm{AZFb}$ region. A common deletion from the AZFa region was observed at locus KALY in $3 / 12(25 \%)$ patients. Deletion of the entire DAZ locus along with DYS240 from AZFc region and DYS237 and DYS236 loci from AZFd region was

Table 1: Frequency of $Y$ microdeletions and chromosome abnormalities/variants in patients with different abnormal sperm parameters.

\begin{tabular}{lcc}
\hline Sperm parameters & $\begin{array}{c}\text { No. } \\
\text { of cases }\end{array}$ & $\begin{array}{c}\text { Y chromosome } \\
\text { microdeletion } / s ~(\%)\end{array}$ \\
\hline $\begin{array}{l}\text { Oligoasthenoterato- } \\
\text { zoospermia (OAT) }\end{array}$ & 73 & $4(5.47 \%)$ \\
Azoospermia (AZ) & 27 & $8(29.63 \%)$ \\
\hline Total & 100 & $12(12.00 \%)$ \\
\hline
\end{tabular}




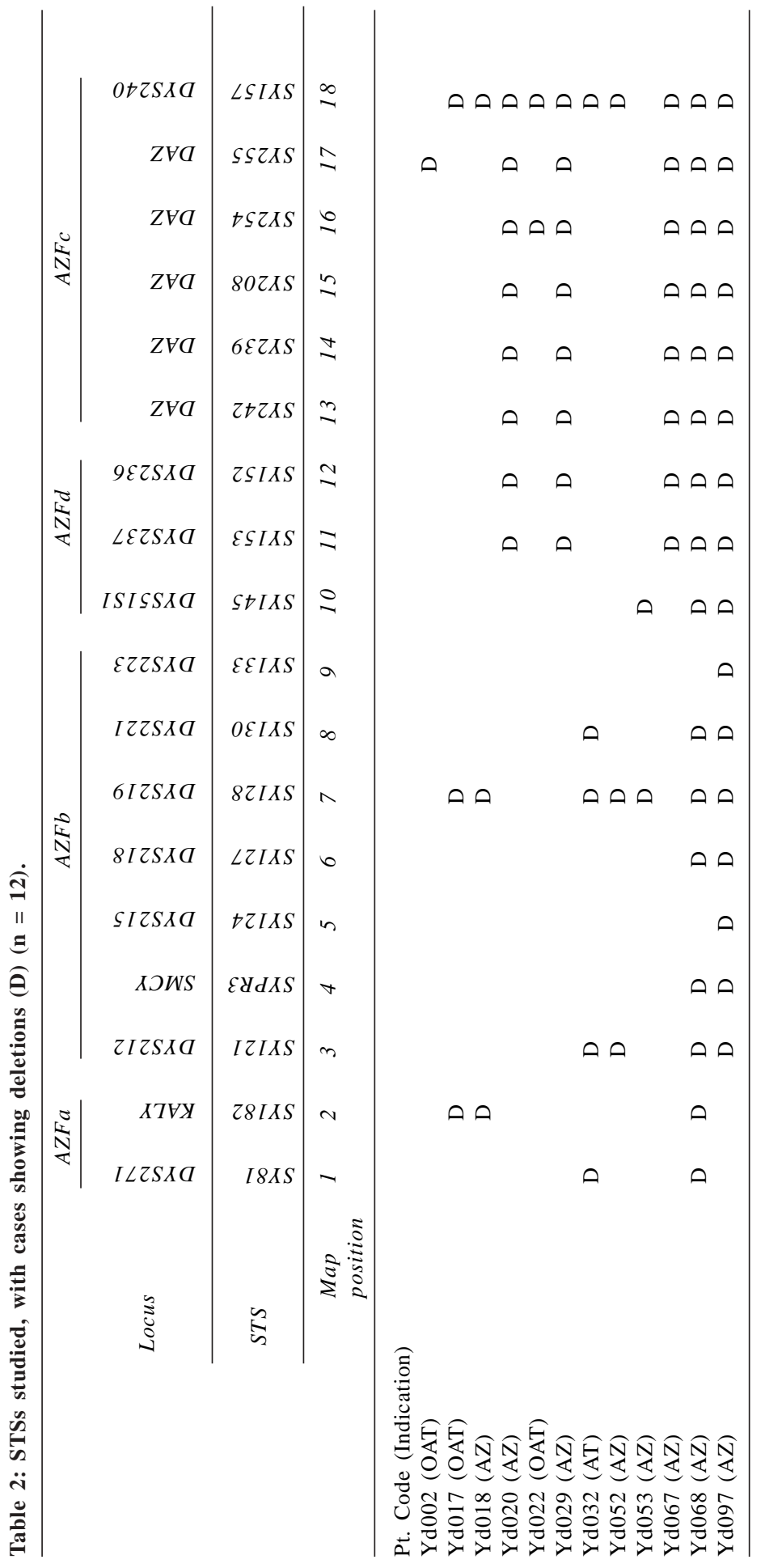


observed in 5/12 (41.67\%) cases.

Y chromosome microdeletions were not detected in the 5 healthy fertile males tested as controls. Electrophoretic analysis of a multiplex PCR showing Y chromosome microdeletions in 2 patients (Yd017 and Yd018) is shown in Figure 1.

\section{DISCUSSION}

Y chromosome microdeletions are known to occur in males with azoospermia and OAT. These infertile males with $\mathrm{Y}$ microdeletions can bear children with the help of ICSI and are at a risk of transmitting the microdeletion to their sons (Cram et al. 2000). They need to be informed and counseled appropriately before starting IVF treatment. The $\mathrm{Y}$ chromosome microdeletions are screened using multiplex PCR whereby several loci are amplified simultaneously in one reaction, thus making it a rapid and convenient method.

The present study compared data on detection of Y chromosome microdeletions and semen analysis of 100 Indian males seeking infertility treatment. Y chromosome microdeletions were seen in 8 of 27 males with azoospermia $(29.63 \%)$ and 4 of 73 with OAT $(5.47 \%)$. Thus the overall frequency of $\mathrm{Y}$ chromosome microdeletion detection in the infertile Indian population in the present study was found to be $12 / 100(12.00 \%)$. The frequency of deletions was much higher in azoospermic males $(29.63 \%)$ compared to cases of OAT $(5.47 \%)$. The proportion of azoospermic males showing the presence of the $\mathrm{Y}$ chromosome microdeletion/s in our study (29.63\%) was higher than that reported by Stuppia et al. (1996) and Yao et al. (2001) as $21 \%$ and $18.7 \%$, respectively.

The DAZ gene cluster localized on the distal

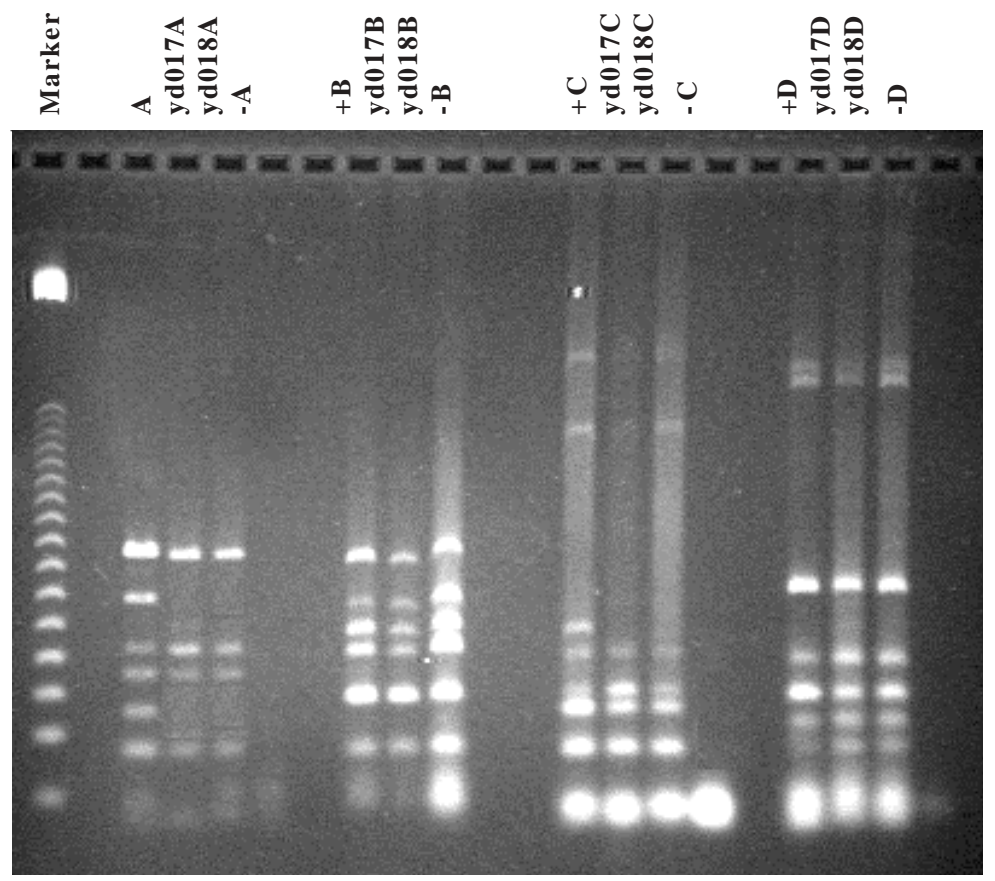

Fig. 1. PCR for Y chromosome microdeletions

Legend:

The picture shows most commonly deleted loci DYS240 from AZFc region, DYS219 from AZFb and KALY from AZFa in 2 patients $\mathrm{Yd} 017$ and $\mathrm{Yd} 018$.

Footnote:

A,B,C,D

$-$

White boxes

Yd017 and Yd018

$=4$ sets of Multiplex MMX

Negative control D/W

Normal control

Deletions

$=$ Test patients 
euchromatic region of the $\mathrm{Y}$ chromosome (AZFc region) is one of the most important candidate genes involved in infertility. Absence of the DAZ gene cluster is known to cause sterility via meiotic arrest or absence of all germ cells (Yao et al. 2001). Kostiner et al. (1998) have reported that about 6$13 \%$ of men with oligospermia or azoospermia showed deletions in all or most DAZ gene clusters. In our study, 5 of $27(18.51 \%)$ patients with azoospermia showed deletions of loci DYS237 and DYS236 from the AZFd region along with deletions of DYS240 and the entire DAZ locus from the AZFc region.

There are some potential problems associated with the use of ICSI in infertile males. Most of the Y chromosome microdeletions may be passed on from father to son and may result in infertility in the son as well. Chang et al. (1999) reported a family with similar DAZ deletions in a father and his four infertile sons. Kent-First et al. (1996) demonstrated that deletions in Yq males could be transmitted to their sons when ICSI was performed. They studied sons born through ICSI in 32 infertile fathers and found 3 affected father/ son pairs where microdeletion of different sizes were found in the $\mathrm{AZFb}$ and $\mathrm{AZFc}$ regions.

It is essential that the infertile male partners are counseled and semen analysis, karyotyping and Y microdeletion studies are done before ICSI is performed. The brothers of infertile males with an abnormal karyotype or Y chromosome microdeletions should also be counseled and analyzed for abnormalities. The Y chromosome microdeletions are likely to be transferred to the sons born through ICSI. PGD can be offered to couples with Y chromosome microdeletions to select unaffected female embryos.

\section{ACKNOWLEDGEMENTS}

The authors would like to thank Jaslok Hospital and Research Centre, for funding from grant RP317.

\section{REFERENCES}

Babu SR, Swarna M, Padmavathi P, Reddy PP 2002. PCR analysis of Yq microdeletions in infertile males, a study from South India. Asian J. Androl, 4(4): 265-268.

Borgaonkar DS, Hollander DH 1971. Quinacrine fluorescence of the human Y chromosome. Nature, 230(5288): 52 .

Chang PL, Sauer MV, Brown S 1999. Y chromosome microdeletion in a father and his four infertile sons.
Hum Reprod, 14(11): 2689-2694.

Cram DS, Ma K, Bhasin S, Arias J, Pandjaitan M, Chu B, Audrins MS, Saunders D, Quinn F, deKretser D, McLachlan R 2000. Y chromosome analysis of infertile men and their sons conceived through Intracytoplasmic sperm injection: Vertical transmission of deletions and rarity of de-novo deletions. Fertil Steril, 74(5): 909-915.

Dada R, Gupta NP, Kucheria K. 2003. Molecular screening for Yq microdeletion in men with idiopathic oligospermia and azoospermia. J Biosci, 28(2): 163168.

Elliott DJ, Cooke HJ 1997. The molecular genetics of male infertility. Bioessays, 19(9): 801-809.

Ferlin A, Moro E, Rossi A, Dallapiccola B, Foresta C 2003. The human Y chromosome's azoospermia factor $\mathrm{b}(\mathrm{AZFb})$ region: sequence, structure and deletion analysis in infertile men. J Med Genet, 40: $18-24$.

Foresta C, Ferlin A, Garolla A, Moro E, Pistorello P, Barbaux S, Rossato M 1998. High Frequency of well-defined Y chromosome deletions in indiopathic Sertoli cell-only syndrome. Hum Reprod, 13(2): 302-307.

Foresta C, Moro E, Ferlin A 2001. Y chromosome microdeletions and alterations of spermatogenesis. Endocrine Reviews, 22 (2): 226-239.

Habermann B, Mi HF, Adelmann A, Bohring C, Backert IT, Kiesewetter F, Aumuller G, Vogt PH 1998. DAZ (deleted in azoospermia) genes encode proteins located in human late spermatids and in sperm tails. Hum Reprod, 13(2): 363-369.

Kent-First M, Kol S, Muallem A, Ofir R, Manor D, Blazer S, First N, Itskovitz-Eldor J 1996. The incidence and possible relevance of Y-linked microdeletion in babies born after intracytoplamic sperm injection and their infertile fathers. Mol Hum Reprod, 2(12): 943-950.

Kent-First M, Muallem A, Shultz J, Pryor J, Roberts K, Nolten W, Meisner L, Chandley A, Gouchy G, Jorgensen L, Havighurst T, Grosch J 1999. Defining regions of the Y-chromosome responsible for male infertility and identification of a fourth AZF region (AZFd) by Y-chromosome microdeletion detection. Mol Reprod Dev, 53(1): 27-41.

Kostiner DR, Turek PJ, Reijo RA 1998. Male infertility: Analysis of the markers and genes on the human Y chromosome. Hum Reprod, 13(11): 3032-3038.

Lilford R, Jones AM, Bishop DT, Thornton J, Mueller R 1994. Case-control study of whether subfertility in men is familial. $B M J, \mathbf{3 0 9}(\mathbf{6 9 5 4})$ : $570-573$.

Pryor J L, Kent-First M, Muallem A, Van Bergen AH, Nolten WE, Meisner L, Roberts KP 1997. Microdeletions in the $\mathrm{Y}$ chromosome of infertile men. N. Engl J Med, 336(8): 534-553.

Raman R 1999. Y chromosome microdeletion in idiopathic male infertility. In: Indo-European Seminar cum workshop on Advances in Human Genetics, 101-102.

Reijo R, Lee TY, Salo P, Alagappan R, Brown LG, Rosenberg M, Rozen S, Jaffe T, Straus D, Hovatta O 1995. Diverse spermatogenic defects in humans caused by Y-chromosome deletions encompassing a novel RNA-binding protein gene. Nat Genet, 10(4): $383-393$ 
Simoni M, Kamischke A, Nieschlag E 1998 Jul. Current status of the molecular diagnosis of Y-chromosomal microdeletions in the work-up of male infertility. Initiative for international quality control. Hum Reprod, 13(7): 1764-1768.

Stuppia L., Mastroprimino G, Calabrese G, Peila R, Tenaglia R, Palka G 1996. Microdeletions in interval 6 of the Y chromosome detected by STS-PCR in 6 of 33 patients with idiopathic oligo or azoospermia. Cytogenet Cell Genet, 72(2-3): 155-158.

Swarna M, Babu SR, Reddy PP 2003. AZFc deletions in idiopathic infertile males from south India. Int $J$ Hum Genet, 3(1): 1-4.

Thangaraj K, Gupta NJ, Pavani K, Reddy AG, Subramanian S, Ram, DS, Ghosh B, Chakravarty B, Singh L 2003. Y chromosome deletions in azoospermic men in India. $J$ Androl, 24(4): 588597.

Tiepolo L, Zuffardi O 1976. Localization of factors controlling spermatogenesis in the nonfluorescent portion of the human $\mathrm{Y}$ chromosome long arm. Hum Genet, 34(2): 119-124.

Vergnaud G, Page DC, Simmler MC, Brown L, Rouyer F Noel B, Botstein D, de la Chapelle A, Weissenbach J 1986. A deletion map of the human Y chromosome based on DNA hybridization. Am $J$ Hum Genet, 38: 109-124.

Vogt P, Chandley AC, Hargreave TB, Keil R, Ma K, Sharkey A 1992 Jul. Microdeletions in interval 6 of the $\mathrm{Y}$ chromosome of males with idiopathic sterility point to disruption of AZF, a human spermatogenesis gene. Hum Genet, 89(5): 491-496.

Vogt PH, Edelmann A, Kirsch S, Henegariu O, Hirschmann P, Kiesewetter F, et al. 1996. Human Y chromosome azoospermia factors (AZF) mapped to different subregions in Yq11. Hum Mol Genet, 5(7): 933-943.

Yao G, Chen G, Pan T 2001. Study of microdeletions in the Y chromosome of infertile men with idiopathic oligo-or azoospermia. J Assist Reprod and Genet, 18(11): $612-616$. 